\title{
Photoelectron-photoion momentum spectroscopy as a clock for chemical rearrangements: Isomerization of the dication of acetylene to the vinylidene configuration.
}

\author{
T.Osipov ${ }^{1}$, C.L.Cocke ${ }^{1}$, M.H.Prior ${ }^{2}$, T.Weber ${ }^{3}$, O.Jagutzki ${ }^{3}$, L.Schmidt ${ }^{3}$, H.Schmidt- \\ Boecking $^{3}$, R.Doerner ${ }^{3}$, A.Landers ${ }^{4}$ \\ ${ }^{1}$ J.R.Macdonald Laboratory, Physics Dept., Kansas State University, Manhattan, KS 66506 \\ ${ }^{2}$ Lawrence Berkeley National Laboratory, Berkeley, CA, 94720 \\ ${ }^{3}$ Institut fuer Kernphysik, University Frankfurt, August-Euler-Str.6,60486,Frankfurt, Germany \\ ${ }^{4}$ Dept. of Physics, Western Michigan University, Kalamazoo, MI
}

\begin{abstract}
We have used complete correlated momentum mapping of the photoelectron and heavy ion products from the dissociation of the dication of acetylene, induced by photoionizing the carbon $\mathrm{K}$ shell of one of the atoms, to map out the angular correlation between the electron and the axis of the target molecule. The (quasi-) symmetric decay is found to proceed through both acetylene and vinylidene configurations. By using the strongly peaked photoelectron emission to "start a clock", an upper limit of 60 fs is placed on the isomerization time from the acetylene to the vinylidene configuration.
\end{abstract}

The importance of the vinylidene configuration of $\mathrm{C}_{2} \mathrm{H}_{2}$ as an intermediate in combustion and other organic reaction pathways has been heavily studied and discussed in the literature for more than two decades since the discovery of this molecule. [see refs. 1-6 for an entry to the literature]. The isomerization between the acetylene and vinylidene configurations of $\mathrm{C}_{2} \mathrm{H}_{2}$ has been heavily studied in both neutral ${ }^{2-6}$ and ionic species ${ }^{7}$ and for numerous excitation/ionization entrance channels. Experiments which provide direct measurements of the time scale for the rearrangement are $\operatorname{rare}^{7}$.

The species under investigation in this paper is the dication of acetylene/vinylidene, which has been comprehensively investigated through various means ${ }^{8-14}$ . In particular, the photo fragmentation of acetylene in the UV region by Thissen et al. $^{14}$, who studied photodouble ionization of acetylene near threshold (35$65 \mathrm{eV}$ ), showed a strong population of the $\mathrm{CH}_{2}^{+}-\mathrm{C}^{+}$ dissociation channel, indicating that the excited molecule often rearranged to the vinylidene configuration following photoionization but prior to dissociation. An accompanying theoretical study in that work, and a following comprehensive $a b$ initio study of the $\mathrm{C}_{2} \mathrm{H}_{2}{ }^{++}$molecule by Duflot et al. ${ }^{15}$, provided a very detailed interpretation of the experimental data and description of the decay pathways for this isomerization as well as for other photodissociation channels. Gadea et al. ${ }^{16}$ suggested that an acetylene molecule with a localized carbon $\mathrm{K}$ hole, produced via $\mathrm{X}$ ray excitation, would be unstable toward the vinylidene configuration, since the hydrogen atom on the side of the localized hole prefers energetically to be on the other end of the molecule. They suggested that the molecule might reconstruct on a time scale comparable to that for the Auger relaxation of the localized hole, and that such a phenomenon might be quite general in nature.

The present article deals with the double ionization of acetylene through photoionization of the molecule just above the carbon K edge (photoelectron energy $10 \mathrm{eV}$ ). The molecule so ionized then populates the dication via Auger electron emission and subsequently fragments into two charged pieces. We measure the momenum vectors of the photoelectron and the emitted fragments. The usual approach ${ }^{17-19}$ is to use the molecular breakup in the radial dissociation approximation to a posteriori align the molecule, and use this to measure the photoelectron angular distribution relative to the molecular axis. In this paper we approximately turn this idea around: we use the photoelectron distribution to indicate the alignment of the target molecular axis at time of photoionization, and then ask to what extent the molecule decays along this axis. For the acetylene configuration (A), we find that the symmetric dissociation into the $\mathrm{CH}^{+}-\mathrm{CH}^{+}$channel is along the line of the target. For the vinylidene final configuration (V), indicated by decay into the $\mathrm{CH}_{2}^{+}-\mathrm{C}^{+}$channel, considerable rotation of the $\mathrm{C}=\mathrm{C}$ bond prior to dissociation is seen. From the quantitative analysis of the data, we deduce the rotation angle and are able to place an approximate upper limit on the time taken by the molecule to rearrange from the $\mathrm{A}$ to the $\mathrm{V}$ configuration.

The experimental arrangement is similar to that described in refs. 17-19, where more detail can be 
found. Linearly polarized (polarization: $\mathrm{x}$-axis; propagation: z-zxis) $310 \mathrm{eV}$ photons from the ALS (beamlines 9.3.2 and 4.0) were incident on a supersonic jet (y-axis) of acetylene gas and ionized the $\mathrm{K}$ shell of one of the carbon atoms of the molecule, ejecting a photoelectron of $10 \mathrm{eV}$. The molecule subsequently Auger decays to the dication and dissociates, usually emitting two singly charged ions. The two ions and the electrons were collected by a static electric field of 10 $\mathrm{V} / \mathrm{cm}$, oriented perpendicular to the photon beam ( $\mathrm{x}-$ axis), and projected onto the faces of position- and timesensitive channelplate detectors equipped with delay-line anodes. From the times and positions with which the particles hit the detectors, the vector momenta of all three particles were reconstructed on an event-by-event basis.

The emission of the photoelectron is a fast process: a $10 \mathrm{eV}$ electron will traverse a typical molecular distance of 2 a.u. in a time of $6 \times 10^{-17} \mathrm{sec}$., during which the atomic constituents of the molecule cannot move nor can the molecule Auger decay. Thus the photoelectron is prompt, and the angular distribution of the photoelectrons with respect to axis of the target molecule is determined before the breakup occurs. The molecule then undergoes an Auger decay in approximately $7 \mathrm{fs}$, somewhat faster than either the dissociation or rearrangement times, and finally dissociates. (In further discussion we will make the simplifying assumption that the Auger decay precedes the dissociation and the rearrangement, although this assumption is not essential to the final conclusions of the paper.)

The photoion-photoion (PIPICO) spectrum (not shown), in which the flight time of the first heavy fragment is plotted versus that of the second one, is only sufficient in our case to isolate decays in which a mass26-amu molecule decays into nearly symmetric pairs $(13 / 13$ or $12 / 14)$, but not to separate these two possibilities. The presence of both of these channels is seen by looking at the calculated fragment momenta in the xz plane. The upper panel of Fig 1. shows the $p_{x}-p_{z}$ momentum spectrum of the mass-26 events, where the momenta are calculated assuming a fully symmetric 13/13 breakup into $\mathrm{CH}^{+}$and $\mathrm{CH}^{+}(\mathrm{A})$. If a single kinetic energy release (KER) were populated in the decay (which experimentally is observed to be nearly the case here, very different from the situation seen in refs. 1719) the events should lie on the heavy black circle. The presence of events on the other two (distorted) dashed light circles is due to the 12/14 breakup of the molecule into $\mathrm{C}^{+}$and $\mathrm{CH}_{2}^{+}(\mathrm{V})$. The presence of two such circles is due to the two possibilities of 12 or 14 hitting as the first event. The observed KER values are 5 and $4.5 \mathrm{eV}$ for the $\mathrm{A}$ and $\mathrm{V}$ channels respectively. By using the fact that the vector sum of the momenta of the two ions must be zero, we are able to select windows in fig. 1 which separate the $\mathrm{V}$ and $\mathrm{A}$ channels if the molecular axis is within 45 degrees of the $\mathrm{x}$ axis; we restrict further discussion to this truncated data set.The $\mathrm{V}$ and $\mathrm{A}$ channels are roughly equally populated. For comparison, we show in the lower panel of fig. 1 what the spectra look like when the complication of the two breakup paths is not present. This panel shows the corresponding data for an ethylene target, where only the fully symmetric $\mathrm{CH}_{2}^{+} / \mathrm{CH}_{2}^{+}$channel is observed.

In fig. 2a we show the angular distribution of photoelectrons measured relative to the molecular dissociation axis, for the A decay channel. The strongly peaked and characteristic f-wave structure is typical of that seen near the shape resonance in $\mathrm{N}_{2}$ and $\mathrm{CO}$, and also appears here. The sharpness of the structure around 45 degrees indicates that the molecule has decayed promptly so that the axis of the decay fragments coincides quite accurately with that of the target. For example, a molecule dissociating into two symmetric fragments along a Coulomb potential curve, starting at an internuclear separation distance of 1.4 a.u. would rotate only 1.4 degrees if the rotational temperature of the target were $80 \mathrm{~K}$. If the dissociation were slow (lifetime more than a hundred fs or so) so that the molecule could rotate appreciably before it came apart, the correlation between photoelectron and photofragment decay axis would become progressively lost. This effect was seen for the CO molecule in ref. 18 .

Fig. $2 b$ shows the corresponding photoelectron angular distribution for the $\mathrm{V}$ channel. The angular distribution is now considerably washed out, a result which we attribute to rotation of the molecule prior to dissociation. In fig. 2c we show for comparison the "singles" laboratory angular distribution of all photoelectrons, with no condition on the molecular axis. This spectrum is nearly the same as that which would be obtained if the molecule rotated for a very long time before it came apart. (We note that this statement is not automatically true, but we verified that it holds in this case by tests done with the ethylene target data.) Fig. $2 b$ is clearly intermediate between $2 \mathrm{a}$ and $2 \mathrm{c}$, indicating some but not complete washout. (The distributions of fig.2 have been forced to be symmetric about the horizontal and vertical axes, as is required by the experimental geometry for the situations of figs. $2 \mathrm{a}$ and $2 c$. For $2 b$, symmetry about the vertical axis is not required in principle, but is expected on physical grounds ${ }^{19}$ and was observed to hold within experimental error, so we chose to symmetrize this distribution as well.)

We have deduced an experimental "rotation angle" from the data of fig. $2 b$ in the following way. It was assumed that the molecule started with a photoelectron distribution of fig. 2a, but before it emitted the heavy fragments it rotated by an angle $\theta$. Using the A channel data, the angles of all particles were calculated in the usual way, but, before plotting, a 
randomization angle, chosen to be random in direction and of a magnitude reflecting a Gaussian distribution centered at $\theta_{0}$, was added to the angle between the photoelectron and the molecular decay axis. This is approximately what the molecule itself does in the decay process. The parameter $\theta_{0}$ was then varied until the distribution of fig. $2 b$ was produced from the data of fig. $2 \mathrm{a}$. The result is shown as a solid curve in fig. $2 \mathrm{~b}$. A plot of the chi-squared of the resulting fit to the data of fig. $2 b$ is shown in fig. 3 as a function of $\theta_{0}$. A clear minimum is seen near $\theta_{0}=0.35$ radians.

It is tempting to directly interpret this angle as due to the product of the initial angular velocity of the molecule, caused by the finite rotational velocity of the target, and the time taken by the molecule to reach the $\mathrm{V}$ configuration. However, it is important to remember that some rotation of the angle of the $\mathrm{CC}$ bond inevitably accompanies the rearrangement from the $\mathrm{A}$ to the $\mathrm{V}$ configuration, even for an instantaneous rearrangement. The minimum rotation consistent with the mass rearrangement depends on the path the $\mathrm{H}$ atom follows in proceeding from its initial position on one end of the molecule to its final home at the other end of the molecule. If it goes straight down the molecular axis, no rotation is required; however, this is a very unlikely path. The details of the transition states through which the molecule is expected to isomerize are described by Duflot et al. ${ }^{15}$, and resemble more closely a tangential path around the center of mass of the molecule. Taking a constant radius path around the center as a model, one can deduce that the minimum rotation of the $\mathrm{CC}$ bond consistent with the mass rearrangement is 0.38 radians, essentially equal to the experimentally observed angle. Thus the data are consistent with an instantaneous rearrangement from the $\mathrm{A}$ to the $\mathrm{V}$ configuration. We conservatively estimate that an additional angle of no more than 0.2 radians due to rotation prior to decay would be consistent with the data. The Auger decay of the $\mathrm{K}$ hole emits an electron with a momentum of 4.6 a.u., which, on a lever arm of 0.7 a.u., would impart to the molecule a maximum angular momentum of $3 \mathrm{a}$.u, with an average value near 2 a.u.. If the molecule had 2 a.u. of rotational angular momentum, it would require only about 60 fs to rotate by 0.2 radians. We thus believe that an isomerization time greater than about $60 \mathrm{fs}$ is inconsistent with our data. If an additional contribution to the target rotational velocity due to its rotational temperature were introduced, an even lower time would be deduced. An isomerization time at least this small is consistent with the expected ${ }^{15}$ bending frequencies for the molecule, of order $10^{14} \mathrm{~Hz}$. We therefore conclude that the $\mathrm{H}$ atom makes a very prompt trip around the periphery of the molecule following ionization.

In conclusion, we have observed that the acetylene molecule with a single carbon $\mathrm{K}$ vacancy often rearranges from the acetylene $(\mathrm{A})$ to the vinylidene $(\mathrm{V})$ configuration before dissociating into the (quasi)symmetric mass-conserving channels of the doubly charged molecule. We have used the very peaked angular structure of the photoelectron angular distribution to deduce that this isomerization process must be a prompt one, with no more than about $60 \mathrm{fs}$ delay between photoelectron emission and final dissociation into the $\mathrm{V}$ channel. We suggest that the quantitative use of such photoelectron distributions as a clock for measuring characteristic times for chemical rearrangments could prove of quite general usefulness.

We thank R.W.Field for helpful discussions. This work was supported by Chemical Sciences, Geosciences and Biosciences Division, Office of Basic Energy Sciences, Office of Science, U. S. Department of Energy.

References:

1. A. H. Laufer, J.Chem.Phys.73, 49 (1980); A. Fahr and A.H.Laufer, J.Chem.Phys.83,908 (1985).

2. K.M.Ervin, Joe Ho and W.C.Lineberger, J.Chem.Phys.91, 5974 (1989).

3. T.Carrington,Jr., L.M.Hubbard, H.F.Schaerfer and W.H.Miller, J.Chem.Phys.80,4347(1984).

4. H.K.Srivastava, A.Conjusteau, H.Mabuchi, A.Callegari, K.K.Lehmann, G.Scoles, M.L.Silva and R.W.Field, J.Chem.Phys.113, 7376 (2000).

5.N.Chang,M.Shen and C.Yu, J.Chem.Phys. 106, 3237 (1996).

6. J.Levin, et al., Phys.Rev.Lett. 81, 3347 (1998).

7.M.J.Jensen, et al., Phys.Rev.Lett. 84, 1128 (2000).

8. T.Ast, et al., Org. Mass. Spectrom. 6, 749 (1972).

9. J. R. Appling, et al., Org. Mass. Spectrom. 18,282 (1983).

10. R.R.Rye, T.E.Madey, J.E.Houston and P.H.Holloway, J. Chem. Phys.69, 1504 (1978).

11. M.Thompson, P.A.Hewitt and D.S.Wooliscroft, Anal.Chem.48,1336 (1976).

12. J.Appel, et al., J. Phys. B 7,406 (1974).

13. S.R.Andrews, et al., Chem.Phys. 166,69 (1992).

14. R.Thissen, J.Delwiche, J.M.Robbe, D.Duflot, J.P.Flament, J.H.D.Eland, J.Chem.Phys. 99, 6590 (1993).

15. D.Duflot,J-M. Robbe and J-P.Flament, J. Chem. Phys. 102, 355 (1994).

16. F.X.Gadea, S.Mathieu and L.S.Cederbaum, J.Mol.Structure (Theochem) 401, 15 (1997).

17. A. Landers, et al., Phys.Rev.Lett.87,013002 (2001).

18. Th Weber, et al., J. Phys. B: At. Mol. Opt. Phys. 34

No 18 (28 September 2001) 3669-3678.

19. T. Jahnke, et al., Phys. Rev. Lett. 88, 073002 (2002).

20.B.Kempgens,et al., Phys.Rev.Lett.79, 35 (1997).

21.T.D.Thomas,etal.,Phys.Rev.Lett.82,1120 (1999).

22.N.Haack, et al., Phys.Rev.Lett. 84, 614 (2000). 

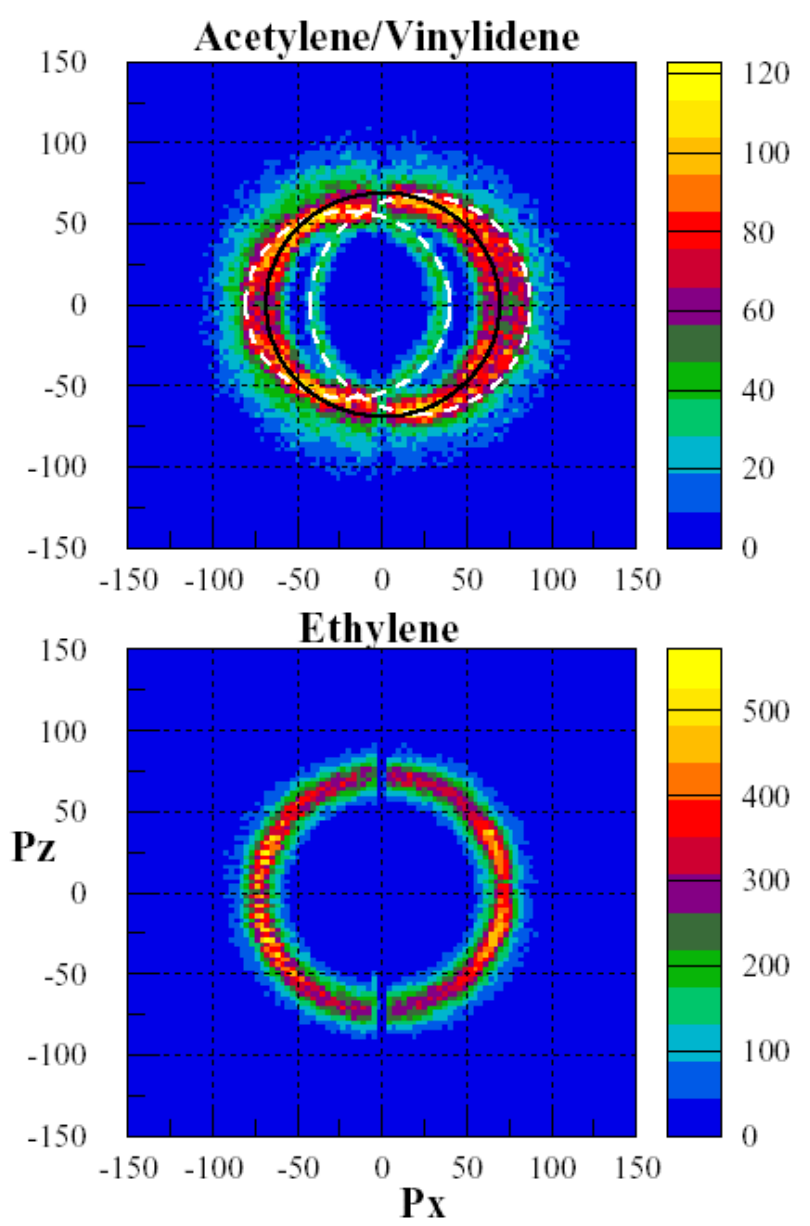

Fig. 1. Transverse momentum spectra calculated from experimental data. The momenta were calculated under the assumption that only the fully symmetric breakup occurs. Upper panel: acetylene target, evaluated under the assumption that only the $\mathrm{CH}^{+} / \mathrm{CH}^{+}$channel is populated. Events on the heavy black circle correspond to this channel. The appearance of the off-center (lightcolor, dashed) distorted circles is due to the breakup into $\mathrm{CH}_{2}{ }^{+}$and $\mathrm{C}^{+}$(see text). Lower panel: corresponding data for an ethylene target, for which only fragmentation into the fully symmetric $\mathrm{CH}_{2}{ }^{+} / \mathrm{CH}_{2}{ }^{+}$is observed.
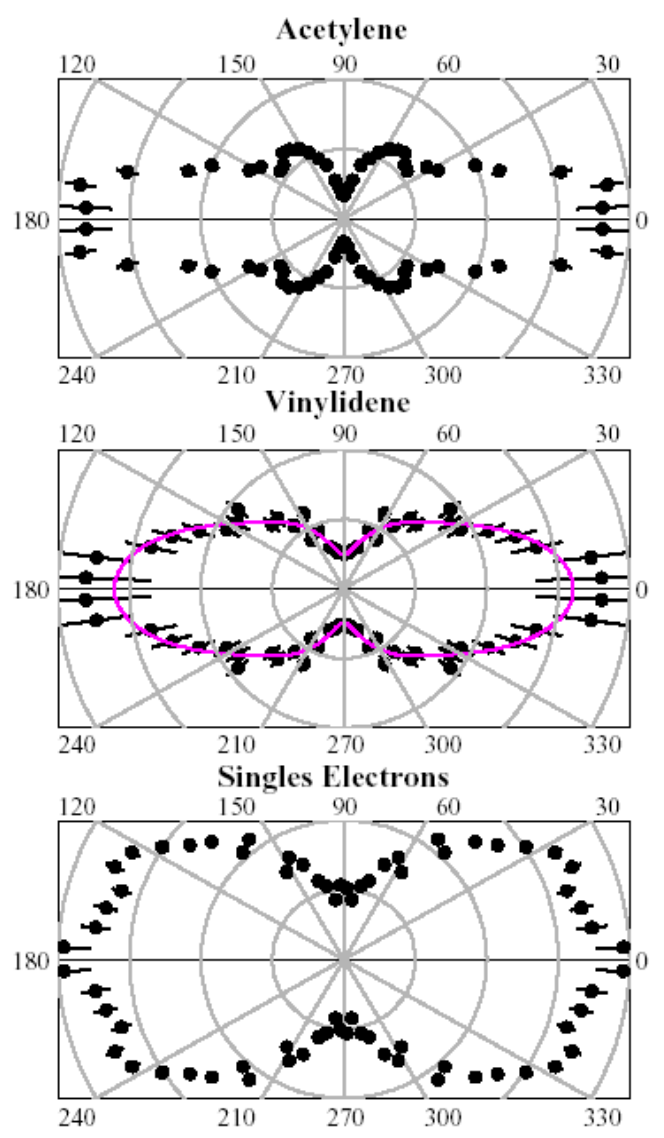

Fig. 2. (a) and (b): Polar plots of photoelectrons in the body-fixed frame for decay through (a) acetylene and (b) vinylidene channels. The molecular fragments are observed along the horizontal axis, and the polarization vector is horizontal. (c) Laboratory "singles" photoelectron distribution. The solid curve in (b) is the fit described in the text.

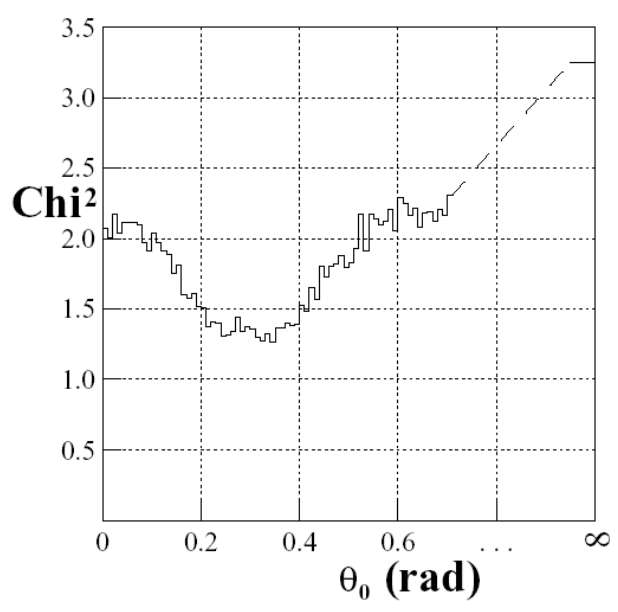

Fig. 3. Chi-squared versus randomization angle $\theta_{0}$, as described in the text. 